\title{
Dynamic behavior of a piezowalker, inertial and frictional configurations
}

\author{
G. Mariotto, ${ }^{\text {a) }}$ M. D'Angelo, and I. V. Shvets \\ Physics Department, Trinity College, Dublin 2, Ireland
}

(Received 17 March 1999; accepted for publication 14 June 1999)

\begin{abstract}
We have examined two configurations of a piezowalker, the inertial and the frictional one, both of which are capable of moving samples with submicrometer steps over a distance of some millimeters. We demonstrated that, contrary to expectations, operation of the frictional walker in which six piezos shear one by one, is still dependent on inertial slip-stick action. We studied the dependence of the step size on the time delay between rising fronts applied to consecutive piezos and we found that the characteristic time associated with the minimum value of the step size is of the order of the time of a sound wave propagating along the rod between the piezos. (C) 1999 American Institute of Physics. [S0034-6748(99)04609-2]
\end{abstract}

\section{INTRODUCTION}

Piezowalkers which can displace objects over distances greater than $1 \mathrm{~mm}$ with submicrometer steps find an increasing number of applications in nanotechnology and scientific instrumentation. They are one of the most important components in scanning probe microscopes where they are commonly used to position a sample under a probe or bring a probe to a sample from a distance of several $\mathrm{mm}$ to the nanometer range. In particular, such piezowalkers are important for designing scanning probe instruments operating in ultrahigh vacuum or at low temperature where operator access to the sample is restricted. Most of these piezowalkers are based on the concept of inertial slip-stick motion. ${ }^{1-4}$ The sample is attached to a platform/rod and moves with steps. The platform/rod rests on a number of piezos under the force of its own weight or under additional force caused by a magnetic clamp or a spring. In a typical arrangement, a flat platform rests on three flat piezoplates placed at the corners of a triangle with equal sides. Often a magnetic clamp is used to hold the platform against the piezos. To achieve this the platform has a hard magnet secured on it, and a soft iron piece is placed in the middle of the triangle formed by the piezos.

To make one step the piezos first deform quickly in one direction and slip under the platform/rod leaving its position virtually unchanged. Then, they deform slowly back simultaneously and drag the platform/rod with them. There is another concept here called "frictional configuration", 5-8 where a rod is held by a number of piezos, and to move it by one step the following is done: first, the piezos deform one by one quickly and slip under the rod leaving its position unchanged. Then, they deform slowly back altogether and drag the rod with them. It is believed that this concept has advantages over the first one as it is not entirely based on inertial action and therefore should be more robust and capable of displacing greater mechanical loads. Some work was carried out on the comparison between the inertial and frictional configuration ${ }^{7}$ which showed that the latter in-

${ }^{a)}$ Corresponding author; electronic mail: MARIOTTG@TCD.IE creases the performance and the reliability of the motor. Nevertheless, it is not clear if the simple models describing their operation are really valid when applied to nanometerrange movements. It is also not clear how big the differences are between these concepts from the performance point of view. These differences become highly important when designing scanning probe instrumentation for operation at low temperature, especially in ultrahigh vacuum (UHV). This is because the piezoelectric coefficients at low temperature decrease and also UHV conditions change the friction properties of surfaces. ${ }^{9}$ From our own experience and correspondence with other groups, we know that the reliability of piezowalkers is often far from sufficient especially at low temperature. As there is often no practical alternative to using a piezowalker for these applications, a better understanding of the physics of the piezowalker is important. In this article, we compare the two concepts of the piezowalker as described above.

\section{PIEZOWALKER DESIGN}

Figure 1 shows a schematic drawing of the top view of the piezowalker used in this experiment. A stainless steel spring plate is screwed on a stainless steel V-shape block to form a triangle. Three rectangular stainless steel blocks are screwed tight on the inner sides of this triangle. On each block, two shearing mode piezoelectric plates are sandwiched between two alumina pads: the lower alumina pads are glued to the block to provide electrical insulation while the upper ones press against a cylindrical sapphire rod. The thickness of the alumina pads is $0.625 \mathrm{~mm}$. The rod is held in place by the force the six alumina pads exert on it. The diameter of the sapphire rod is $6 \mathrm{~mm}$ and its length is 65 $\mathrm{mm}$. The six piezoelectric plates are EBL No. 2 shearing piezos $7 \times 5 \times 1 \mathrm{~mm}$ from Staveley Sensors Inc. ${ }^{10}$ Their piezoelectric coefficient is $d_{15}=5.84 \AA / \mathrm{V}$. Every part of the walker is UHV compatible. A photograph of the piezowalker is shown in Fig. 2.

Especially for the purpose of this study, we have constructed a very versatile electronic signal generator to actuate 


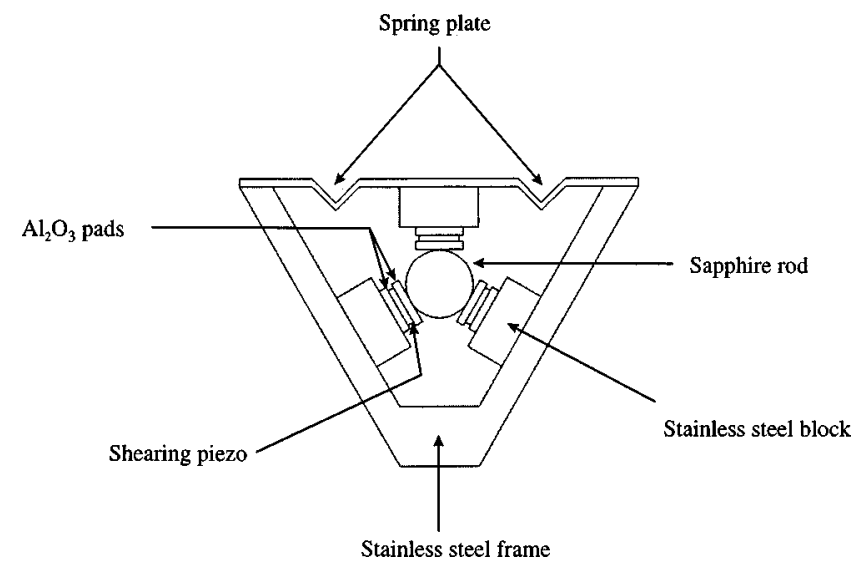

FIG. 1. Schematic of the top view of the piezowalker.

the piezos. The generator consists of a fast digital-to-analog board the output of which is connected to the input of a high voltage amplifier (HVA). The board in turn is connected to a $\mathrm{PC}$ and it therefore can generate output analog signal according to parameters determined by an operator. The digital-toanalog board in fact generates six independent signals simultaneously and the HVA also has six independent inputs. Therefore, we can apply signals to six piezos quite independently from each other and can easily vary the form of these signals. For example, we could actuate all the six piezos at the same time using the motor as an inertial walker or apply the signals with a specified delay between the six channels making it work in a frictional configuration. The parameters of the signal generator were as follows. The digital-to-analog board converted the digital signals supplied by the PC into an output voltage in the range of $0-5 \mathrm{~V}$. The board can change the value of the output voltage from 0 to $5 \mathrm{~V}$ in just under $2 \mu$ s (full swing). This time is determined by the slew rate of the analog output front of the board and not by the PC. The HVA have a gain of up to 100 and have the range of the output voltages from 0 to $400 \mathrm{~V}$. The HVA can swing the signal from 0 to $400 \mathrm{~V}$ (full swing) in just under $5 \mu$ s (backward movement) into a capacitive load of some $0.5 \mathrm{nF}$. This load is equivalent to the capacitance of a piezo plate of the walker connected with $1 \mathrm{~m}$ long cable, i.e., most relevant to our experimental situation.

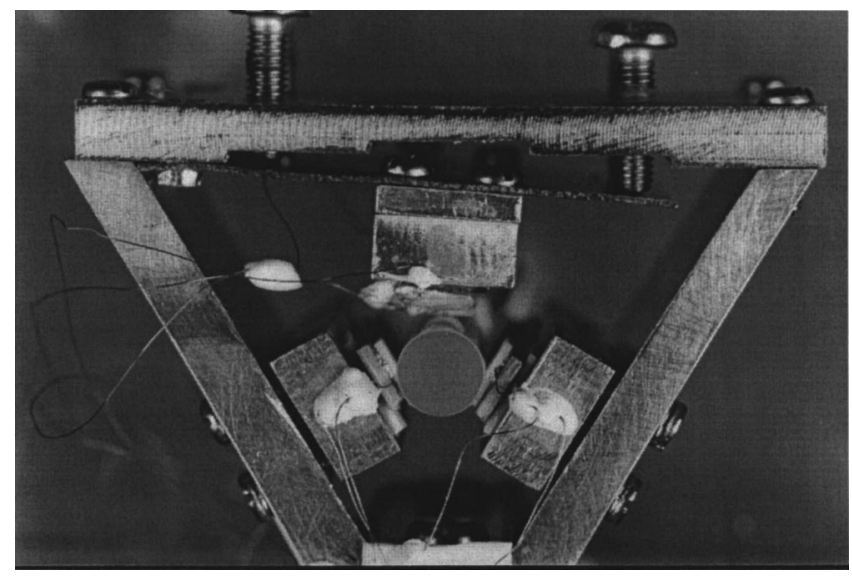

FIG. 2. Photograph of the piezowalker.

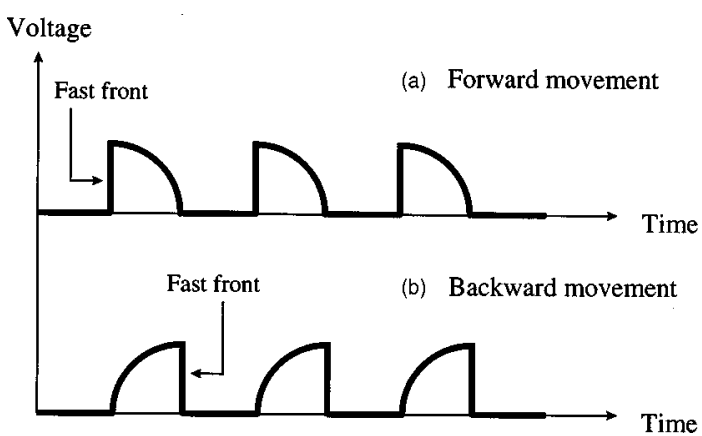

FIG. 3. Quarter sine wave signal applied to achieve (a) forward and (b) backward movement.

\section{PERFORMANCE OF THE PIEZOWALKER}

The first tests carried out on the piezowalker had the purpose of estimating the step size versus the voltage applied to the piezos and establishing the voltage threshold, i.e., the voltage amplitude at which the walker starts making steps. For forward movement, we applied a repetitive signal which is composed of quarter sections of a period of a sine wave [see Fig. 3(a)] and measured the step size against the amplitude of the voltage $V$. For backward movement, the signal was reversed, so that the voltage first slowly increased from 0 to $400 \mathrm{~V}$ and then rapidly changed back to $0 \mathrm{~V}$ [see Fig. 3(b)]. The step size $\alpha=\Delta z /(\Delta t \times \nu)$ was measured in the following way: the walker was run for a given time $\Delta t$ at a frequency $\nu$. The distance $\Delta z$ covered by the walker was then measured by a micrometer fixed under the sapphire rod. In these experiments, the piezos were sheared one by one and the delay between the start of the fast fronts of the signals applied to consecutive piezos was $20 \mu$ s. The step size for both backward and forward movement shows a good linearity with the voltage (see Fig. 4). The maximum step size measured, $1700 \AA$, is consistent with the value of the shear deformation applied to the piezo, $\Delta l=d_{15} V=2300 \AA$ at $V$ $=400 \mathrm{~V}$. As clear from Fig. 4 , there is a difference between the backward and the forward movement. It is not surprising that the step size of the backward movement in which the rod moves down is greater than that of the forward movement in which the rod moves up. There may be an influence of the weight of the rod. However, we have established that such a force should result in a offset of the line for the forward

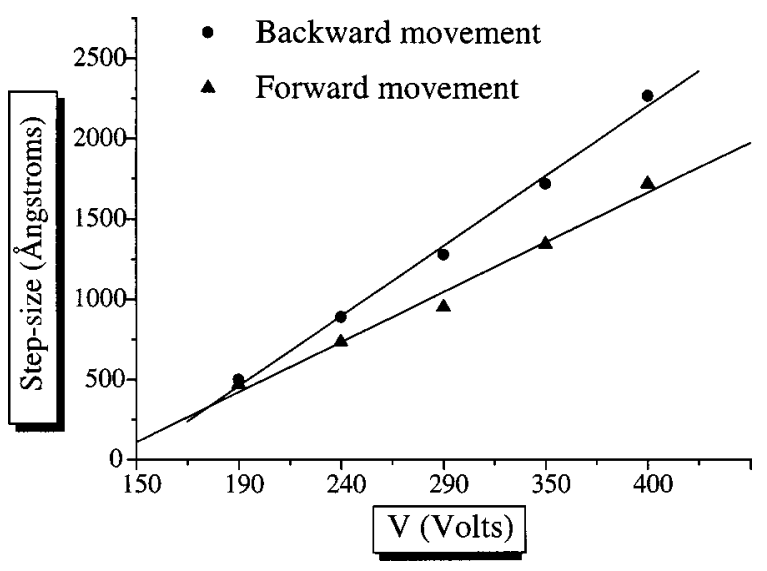

FIG. 4. Step size vs voltage for backward and forward movement. 
movement step size with respect to the line for the backward movement. The two lines should remain parallel to each other and should just be offset along the vertical axis. On the contrary, the two curves in Fig. 4 are not parallel to each other. To exclude effects of gravity on the differences between the lines for forward and backward movements, further tests have been carried out with the walker in the horizontal position. The results obtained do not differ from the ones for the walker in the vertical position; the influence of gravity does not explain the difference between the two curves in Fig. 4. Similar results were obtained by Blackford and Jericho, ${ }^{11}$ they explained the discrepancy between forward and backward steps in terms of surface irregularities or contamination. This argument is not very convincing. We have established that the difference comes from the electronic signal driving the walker. The charging path in the HVA is different from the discharging one. This fact leads to a different duration of the fast front for the forward (charging) and backward (discharging) movements. For the backward movement, the sharp front of the signal does not depend on the amplitude of the wave. On the contrary, the time of the sharp front for the forward signal increases from 3 to $18 \mu$ s when the amplitude of the wave increases from 150 to $400 \mathrm{~V}$. We suggest that as the time of the fast front increases, the piezos do not slide fully under the rod. Instead they may slightly drag the rod with them. From the practical point it may not be correct to consider the rod held immobile by five other piezos as the sixth one moves under the influence of a sharp voltage front. As the fast front is applied consecutively to all the six piezos, the rod could be dragged over a distance $\Delta l^{*}$. Therefore, the step size is reduced from the theoretical value $\Delta l=d_{15} \times V$ by a value $\Delta l^{*}$.

Other tests have been carried out to estimate the influence of the slew rate of the signal on the dynamic behavior of the piezowalker. The aim was to prove that the slew rate is a parameter which plays a critical role in the motion of the sapphire rod, even for the frictional configuration. The rise time of the signal is partly determined by the capacitive load of the piezoelectric to which the signal is applied. Our HVA, whose output impedance is $1.5 \mathrm{k} \Omega$, has a characteristic rise time of less than $5 \mu$ s to ramp the voltage from 0 to $400 \mathrm{~V}$ in the backward mode and less than $20 \mu$ s in the forward mode. The measured capacitance of our piezoelectric plates is about $0.5 \mathrm{nF}$. We expected to see a reduction in the efficiency of the movement as the rise time of the signal increased. To increase the rise time of the signal, we connected capacitors of $1,2.2,4.7$, and $10 \mathrm{nF}$ in parallel with the piezos at each output channel of the HVA. We found that decreasing the slew rate leads to a substantial decrease in the step size and to an increase in the threshold voltage (see Fig. 5). This effect can be understood if we consider the compliance of the piezoplates/alumina pads clamp arrangement. As explained earlier, the alumina pads which press against the sapphire rod are glued onto the shearing piezoplates. Due to the compliance of the glue, which is greater than that of piezoceramic and alumina, part of the force applied to the piezos will be absorbed and not fully transferred to the alumina pads. The compliance of a glue to a force depends on the time characteristic of the force applied. It is clear that compliance to a

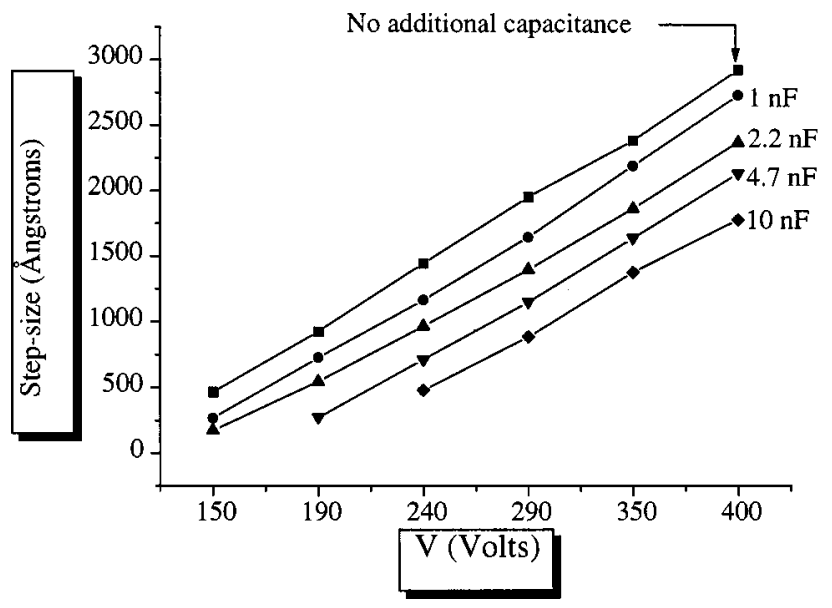

FIG. 5. Step size vs voltage for different values of capacitance added in parallel with the piezos.

static or slowly changing force will be greater than the one for a rapidly changing force which is characterized by a fast slew rate. In the case of a slow rise time of the signals applied to the piezos, it will be more difficult to break the static friction limit with the sapphire rod. As a consequence, the deformation of the layer of glue will be greater, thus leading to a dissipation of the force applied. In the case of a very fast rise time it is easier to overcome the friction limit and then the alumina pads will slip under the sapphire rod. Therefore, as the slew rate decreases, effect of the compliance of the glue leading to a reduction in the step size becomes more noticeable.

A possible way of improving the performance of the piezowalker is therefore to increase the slew-rate of the circuit, i.e., to decrease the rise time of the signals applied to the piezos. It is important to emphasise that these results apply to the frictional piezowalker which is not supposed to be dependent on the inertia. We can therefore conclude that the simple model in which the rod is assumed to be held immobile by five piezos as the sixth one deforms, does not explain the results well. In other words, even if these piezos are deformed one by one, they will not slip under the rod unless the deformation of each piezo is done quickly.

\section{INERTIAL WALKER AND FRICTIONAL WALKER}

Dependence of the step size against the delay time for backward and forward movements at the voltage amplitude of $400 \mathrm{~V}$ is shown in Fig. 6. It is worth noting again that the delay time equal to zero (first point on the graph) corresponds to the inertial mode. The general shape of the dependencies is the same for both backward and forward movements: we have a significant minimum for $5 \mu$ s delay time and then variations with a characteristic time of some $30-40$ $\mu$ s, corresponding to an oscillation frequency of some 33-25 $\mathrm{kHz}$. The variations observed with the characteristic time of some 30-40 $\mu$ s were thought to come from oscillations of the walker itself, whose $\mathrm{V}$-shape is quite similar to a tuning fork. To verify the validity of such an hypothesis, we put an accelerometer on one of the stainless steel plates of the motor. We detected the main resonance vibration frequency at $31 \mathrm{kHz}$, which is consistent with the value taken from the 


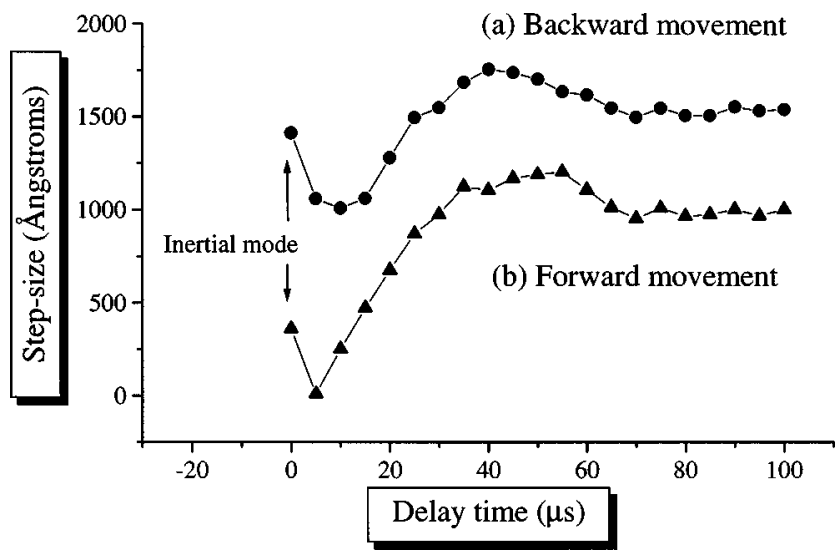

FIG. 6. (a) Step size vs delay time. Backward movement. (b) Step size vs delay time. Forward movement.

step size versus delay time curves. We also detected vibrations at some 44 and $50 \mathrm{kHz}$ with smaller amplitudes, which are thought to correspond to other modes of vibration of the walker. These measurements with the accelerometer also convinced us that the nature of the minimum at $5 \mu \mathrm{s}$ has nothing to do with mechanical vibrations of the V-shape body of the walker. Another thing we excluded as a possible reason for the minimum at $5 \mu \mathrm{s}$ is the rise or fall time of the sharp front of the wave. We were particularly anxious to find out if the minimum was simply due to the sharp front time as it was indeed in the range of some $5-10 \mu$ s, i.e., close to the time of the minimum. To do this, we changed the sharp front time by a factor of 2 or more. This was done by changing the amplitude of the wave in the forward mode from 250 to 400 $\mathrm{V}$. This corresponds, in the forward mode, to a change in the sharp front from 3 to $18 \mu \mathrm{s}$. However, the time corresponding to the minimum remained unchanged at $5 \mu \mathrm{s}$. It is worth noting that for the time delay between the consecutive piezos of less than $4 \mu \mathrm{s}$, the walker did not operate in a pure frictional mode. In other words, the second piezo would start shearing before the first is fully sheared due to the finite time of the fast front. At the same time it is important to emphasise that the minimum at $5 \mu$ s was indeed observed by us also for an amplitude of the wave of $250 \mathrm{~V}$, when the time of the fast front was only $3 \mu$ s, i.e., when the walker did operate in a pure frictional mode.

To explain these results, we propose that when comparing the inertial walker and the walker in the frictional configuration, we should take into account the excitation of the acoustic waves by the rapidly deforming piezos. During the rising phase, the impact force of the piezos against the sapphire rod excites an elastic wave in the rod itself. It propagates along the rod with a velocity given by $\mathrm{v}_{s}=\sqrt{Y / \rho}$, where $Y=3.7 \times 10^{11} \mathrm{~N} / \mathrm{m}^{2}$ and $\rho=3.97 \times 10^{3} \mathrm{~kg} \mathrm{~m}^{3}$ are, respectively, the Young's modulus and the density of the sapphire. The characteristic time required for this wave to cover the distance of some $2 \mathrm{~cm}$ between two consecutive piezos is about $2 \mu$ s. Launching such a wave can reduce the step size. For example, if the direction of the wave in the rod launched by the first piezo coincides with the direction of the shearing of the second piezo, the second piezo will not slide anymore on the rod but will shear with the wave. For this to occur, the wave should arrive to the second piezo exactly at the moment when it is due to shear. This phenomenon will occur three times if the six piezos, are sheared in a sequence: lower piezo-upper piezo etc. It is to be noted that the minimum is highly reproducible and is independent on the wave amplitude or the pressure exerted by the alumina pads on the rod. A wave is also launched through the $\mathrm{V}$-shape body of the walker. The typical propagation time for such a wave is about $4 \mu$ s to cover a distance of $2 \mathrm{~cm}$, as the Young's modulus for stainless steel is $Y=1.9 \times 10^{11} \mathrm{~N} / \mathrm{m}^{2}$ and its density is $\rho=7.96 \times 10^{3} \mathrm{~kg} \mathrm{~m}^{3}$. The timing of the minimum does not change as the pretension of the spring on the rod changes, suggesting that this phenomenon is an intrinsic property of the system characterized only by its size and materials of which the walker is made. We can also see that in the frictional configuration one can achieve a greater value of the step size than in the inertial one if the time interval between the shearing of two consecutive piezos is large enough.

\section{DISCUSSION}

We examined experimentally two important configurations of the piezowalker: the inertial and the frictional one. We demonstrated that even in the frictional configuration the step size and the threshold voltage depend on the rise time of the fast front. Thus, in practice, even in the frictional configuration, the inertia of the rod plays an important role. The walker operating in the frictional configuration is capable of a greater step size and thus is more efficient than the inertial one. However, this is only true when the delay time between the fast fronts applied to different piezos is sufficiently long, longer than $30 \mu$ s for the walker of our dimensions. The step size of the frictional walker decreases to below the value of the inertial one for the delay time of up to $20 \mu$ s and reaches a minimum at 5-10 $\mu \mathrm{s}$. The timing of this minimum does not depend on the mechanical preload applied by the spring on the rod. The characteristic time of this minimum is of the order of the time of a sound wave propagating between the piezos along the rod and the $\mathrm{V}$-shape body of the walker. This suggests that generation of the sound waves by fast deforming piezos must be taken into account when modelling the piezowalker.

\section{ACKNOWLEDGMENTS}

Financial support of the Irish agency Forbairt, Contract No. ST 97/203 is gratefully acknowledged. The authors would like to thank Ciarán Seoighe for his suggestions and Dave Grouse for machining the components of the piezowalker.

${ }^{1}$ D. W. Pohl, Rev. Sci. Instrum. 58, 54 (1987).

${ }^{2}$ Ph. Niedermann, R. Emch, and P. Descouts, Rev. Sci. Instrum. 59, 368 (1988).

${ }^{3}$ J. W. Lyding, S. Skala, J. S. Hubacek, R. Brockenbrough, and G. Gammie, Rev. Sci. Instrum. 59, 1897 (1988).

${ }^{4}$ Ch. Renner, Ph. Niedermann, A. D. Kent, and Ø. Fisher, Rev. Sci. Instrum. 61, 965 (1990) 
${ }^{5}$ S. H. Pan, Piezo-electric Motor, International Patent Publication No. WO 93/19494, International Bureau, World Intellectual Property Organization (30 September 1993).

${ }^{6}$ I. B. Altfeder et al., Rev. Sci. Instrum. 64, 3157 (1993).

${ }^{7}$ A. P. Volodin and A. M. Troyanovskii, Instruments and Experimental
Techniques (Plenum, New York, 1997), Vol. 5, p. 724.

${ }^{8}$ D. Wehnes et al., Appl. Phys. A: Mater. Sci. Process. 66, S41 (1998).

${ }^{9}$ W. Wolfs and A. M. Simpson, Rev. Sci. Instrum. 58, 2193 (1987).

${ }^{10}$ Staveley Sensors, Inc., 91 Prestige Park Circle, East Hartford, CT 06108.

${ }^{11}$ B. L. Blackford and M. H. Jericho, Rev. Sci. Instrum. 61, 182 (1990). 